\title{
Indeterminism, Asymptotic Reasoning, and Time Irreversibility in Classical Physics
}

\author{
Alexandre Korolev ${ }^{1}$
}

\begin{abstract}
A recent proposal of Norton (2003) to show that a simple Newtonian system can exhibit stochastic acausal behavior by giving rise to spontaneous movements of a mass on the dome of a certain shape is examined. We discuss physical significance of an often overlooked and yet important Lipschitz condition the violation of which leads to the existence of anomalous non-trivial solutions in this and similar cases. We show that the Lipschitz condition is closely linked with the time reversibility of certain solutions in Newtonian mechanics and the failure to incorporate this condition within Newtonian mechanics may unsurprisingly lead to physically impossible solutions that have no serious metaphysical implications. To further support this view we also discuss how certain solutions in hydrodynamics associated with first order differential equations (ODEs) with spatially non-Lipschitz right-hand side lead to lack of important properties such as stability with respect to perturbations and Markovianity in time.
\end{abstract}

\section{Introduction}

2 The Mass on the Dome

2.1 Setting the Stage: The Original Formulation

2.2 The Mass on the Dome: Cartesian Perspective

2.3 The Mass on the Dome vs. the Mass on the Pinnacle

\footnotetext{
${ }^{1}$ Department of Philosophy, University of British Columbia, Vancouver, BC, V6T 1Z1, Canada, email: korolev@interchange.ubc.ca.
} 
2.4 The Mass on the Dome or the Mass in the Air?

\subsection{The Mass on the Dome, Modified}

3 The Lipschitz Condition

4 Asymptotic Reasoning and Time Irreversibility

4.1 Models and Idealizations

4.2 Stability and Parameter Sensitivity

4.3 The Mass on the Dome and Time Irreversibility

5 Lipschitz Indeterministic Solutions and the Markov Condition

5.1 Generalized Flows in Hydrodynamics

5.2 Non-Lipschitz Velocity Fields, Regularizations, and the Markov Condition

\section{Introduction}

Abstruse theories like quantum mechanics and general relativity routinely violate common intuitions about causality and determinism. In contrast, classical physics is often assumed to be a paradigm example of a fully deterministic physical theory that never violates these intuitions, or that violates them only in the most extreme circumstances which render such situations as plainly unphysical. A number of authors have argued that this is not so, and that classical physics is a poor choice of hunting ground for such beliefs. A definitive guide to the discussion is John Earman's A Primer on Determinism (1986) that collects and discusses various situations that threaten uniqueness of solutions for common differential equations governing dynamics of ordinary classical systems. A more recent attempt by John Norton (2003) presents another simple Newtonian system that seems to exhibit stochastic acausal behavior in that it allows generation of spontaneous motion of a mass without any 
external intervention or any change in the physical environment. The latter system is of particular interest since, unlike most of Earman's examples, it does not seem to involve, at least directly, any singularities, wild divergences, or any other bullying with infinities of physically meaningful parameters in any way that often leave the true believer of determinism unsatisfied. Norton uses this example to support his vision of causality as a notion belonging more in folk science rather than being a fundamental principle underlying all natural processes and unifying all the domains of science at some deeper level.

While by and large sympathetic with the general thrust of the "anti-fundamentalist" program of Norton, we intend to demonstrate that his mass on the dome example fails to provide support for such a view. We show that the existence of anomalous non-trivial solutions in this case is due to the violation of an often overlooked and yet important Lipschitz condition and discuss its physical significance. We show that the Lipschitz condition is closely linked with several important temporal properties of certain solutions such as time reversibility and Markovianity in time so that the failure to recognize this condition as an important implicit assumption within Newtonian mechanics may unsurprisingly lead to physically impossible solutions that have no serious metaphysical import, as, for instance, in Norton's causal skeptical anti-fundamentalist program.

The rest of the paper is organized as follows. Subsection 2.1 introduces Norton's mass-on-the-dome example and the time reversal argument. In Subsections 2.2-2.5 we expose the loopholes of this example, some of them potentially fatal to the project, and introduce a cleaner version free of loopholes and unnecessary complications. Section 3 discusses the Lipschitz condition as it appears in the theory of ordinary differential equations (ODEs) and as it enters the mass-on-the-dome example. Section 4 applies (infinite) asymptotic reasoning to display the asymmetry of time-reversal arguments for the 
non-Lipschitz velocity fields and an unphysical singular nature of the spontaneous motion generation is revealed. In Section 5 we draw several results from fluid dynamics to further illustrate how certain solutions associated with first order differential equations with spatially non-Lipschitz right-hand side may lead to lack of important properties such as stability with respect to perturbations and Markovianity in time.

\section{The Mass on the Dome}

\subsection{Setting the Stage: The Original Formulation}

A unit point mass slides frictionlessly on the surface under the action of gravity. The surface is shaped as a symmetric dome described by the equation:

$$
y(r)=-\frac{2}{3 g} r^{3 / 2}
$$

where $r$ is the radial coordinate in the surface, i.e., the distance traveled by the mass from the highest point of the dome along the surface, $|y|$ specifies how far the dome surface lies below the apex as a function of $r$, and $g$ is the acceleration due to gravity (Fig. 1). 


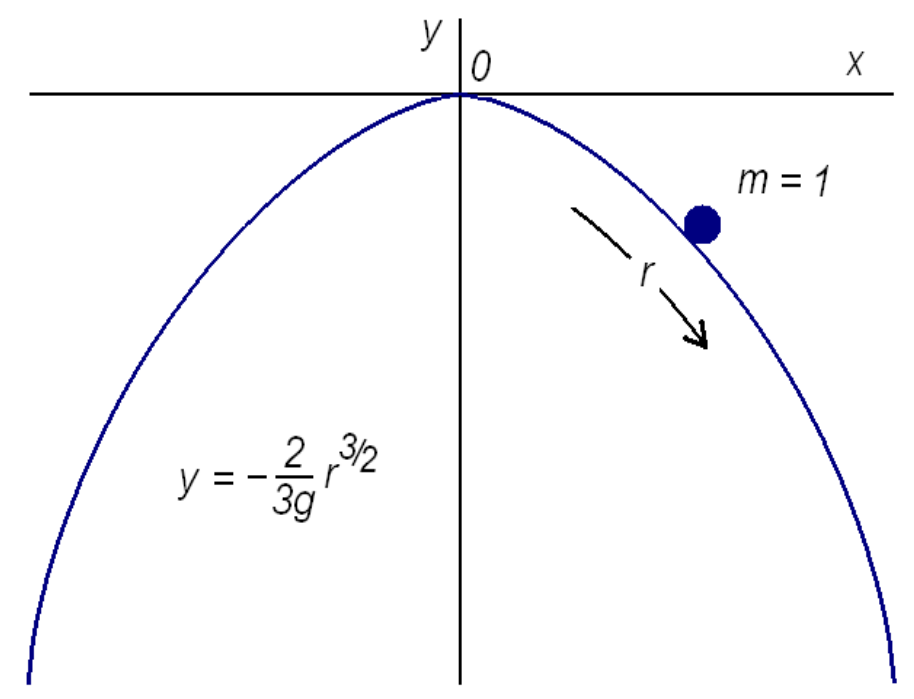

Fig. 1 Mass sliding on a dome.

At any point, the magnitude of the gravitational force tangential to the surface is $F_{\tau}=-\frac{d(g y)}{d r}=r^{1 / 2}$ and is directed outward. Newton's second law of motion, $F=m a$, applied to the mass on the surface gives

$$
\frac{d^{2} r}{d t^{2}}=r^{1 / 2}
$$

If the mass is initially located at rest at the apex $r=0$, then one obvious solution to (1) for all times $t$ is a trivial one:

$$
r(t)=0 .
$$

The mass simply remains at rest for all times. However, there exists another large class of unexpected solutions. For any radial direction,

$$
r(t)=\left\{\begin{array}{l}
\frac{1}{144}(t-T)^{4}, \text { for all } t \geq T \\
0, \text { for all } t \leq T
\end{array}\right.
$$


where $T \geq 0$ is an arbitrarily chosen constant. By direct computation one can readily confirm that (4) satisfies Newton's second law (2).

Note that equation (4) describes a point mass sitting at rest at the apex of the dome, whereupon at an arbitrary time $T \geq 0$ it spontaneously moves off in some arbitrarily chosen radial direction.

The solutions (4) appear to be fully in accord with Newton's second and first laws, if one takes the first law in its instantaneous form as follows:

In the absence of a net external force, a body is unaccelerated.

Indeed, for all times $t<T$, there is no net force applied, since the body is at position $r=0$, the force free apex; and the mass is unaccelerated.

For all times $t>T$, there is a non-zero net force applied, since the mass is at positions $r>0$ not the apex, the only force free point on the dome; and the mass accelerates in accord with $F=m a$.

Finally, when $t=T$, the direct computation of the mass acceleration from the equation (4) gives us

$$
a(t)=\left\{\begin{array}{l}
\frac{1}{12}(t-T)^{2}, \text { for all } t \geq T \\
0, \text { for all } t \leq T
\end{array},\right.
$$

so that at $t=T$, the mass is still at the force-free apex $r=0$ and the mass acceleration $a(0)$ is equal to zero. Again, no force, no acceleration, exactly as the first law requires.

What about the initiating cause that sets the mass in motion in the first place? Surely the instant $t=T$ is not the first instant at which the mass moves; it is the last instant at which the mass does not move. In fact, one can name no first instant at which the mass 
moves. So, if there is no first instant of motion, then there is no first instant at which to seek the initiating cause.

Yet another powerful argument can be given in support of acausality of the mass motion. This argument involves the time reversal trick. Since the Newtonian dynamical laws of gravitational systems are invariant under time reversal we can invert the slidingdown-the-dome scenario to produce another legitimate solution which insults the principle of causality. Instead of having the mass starting at the apex of the dome, we will imagine it starting at the rim and that we give it some initial velocity directed exactly at the apex. If we give it too much initial velocity, it will pass right over the apex to the other side of the dome. If we give it too small initial velocity, it will rise toward the apex, but before it reaches the apex it halts and slides back to the rim. Now if we give the mass just the right amount of initial velocity, it will rise up and momentarily halts exactly at the apex.

The proper mathematical analysis of the latter situation reveals that the time required for the mass to reach the apex moving along the surface of this particular shape is finite. That this time is finite is essential for the time reversal trick to succeed. Infinite time would mean that the mass never actually arrives at the apex, and the time reversal scenario would display a mass that has been in motion at all past times, without any spontaneous launches. It should be emphasized that by no means this feature is common to all domes. For hemispherical or parabolic domes, for instance, the time taken for the mass to reach the apex to its momentary halt is unbounded. In the case of the dome of Fig. 1 the time reversal trick does work.

\subsection{The Mass on the Dome: Cartesian Perspective}


Defining the surface by $y(r)=-\frac{2}{3 g} r^{3 / 2}$, in terms of the distance $r$ traveled by the mass along the surface, conceals important details about the actual geometry of the dome and directionality of motion as viewed from the "external" Cartesian perspective. Special care should be taken not to overlook these details since they may prove crucial in the general case. Indeed, as the present section shows, the original formulation of the mass-on-thedome example harbors several loopholes, some of which potentially fatal to the project. Fortunately, most of these difficulties are reparable and can be overcome by slightly modifying the original formulation of the problem. As the next section shows, the modified version inherits all the strangeness of being a source of spontaneous motion generation without our having to deal with the loopholes and unnecessary qualifications.

Consider an axially symmetric dome, and $x$ is a Cartesian radial variable. In what follows, we consider only non-negative $x$ 's, and then extend the results to incorporate the negative values of the $x$-coordinate. For the curvilinear coordinate $r$ measured along the surface slice cut vertically through the apex at the origin we have

$$
\frac{d r}{d x}=\sqrt{1+\left[y^{\prime}(x)\right]^{2}}
$$

so that

$$
\frac{d y(r)}{d r}=-\frac{1}{g} r^{1 / 2}=\frac{d y}{d x} \cdot \frac{d x}{d r}=\frac{y^{\prime}(x)}{\sqrt{1+\left[y^{\prime}(x)\right]^{2}}}, y^{\prime}(x) \leq 0
$$

Expressing the coordinate $r$ we can write down

$$
r=g^{2} \frac{\left[y^{\prime}(x)\right]^{2}}{1+\left[y^{\prime}(x)\right]^{2}} .
$$

Putting $v=y^{\prime}$ and differentiating both sides of (6) gives us 


$$
\frac{d v}{d x}=\frac{1}{2 g^{2}} \frac{\left(1+v^{2}\right)^{5 / 2}}{v}, \text { so that } \int \frac{v d v}{\left(1+v^{2}\right)^{5 / 2}}=\frac{1}{2 g^{2}} x+\text { Const }
$$

Integrating the left-hand side of the last expression we get:

$$
\left(1+\left[y^{\prime}(x)\right]^{2}\right)^{3 / 2}=\frac{1}{C-k x}
$$

for $k=\frac{3}{2 g^{2}}$ and some constant $C$.

The first observation to make is that the dome surface appears to be defined not for all $x$ 's, but only for $x^{\prime}$ s out of some (final) interval $[0, L)$. Indeed, the left-hand side of (7) is always greater or equal to 1 , so it must be the case that $0 \leq C-k x \leq 1$ for all $x$ out of some interval $[0, L)$. That provides the constraint on the possible values the constants $C$ and $L$ can take:

$$
0<k L \leq C \leq 1 \text {, where } L<1 / k \text {. }
$$

Finally, integrating (7) and incorporating negative values of $x$, we can express $y(x)$ as a function of the Cartesian coordinate $x$ :

$$
y(x)=-\frac{1}{k}\left[1-(C-k|x|)^{2 / 3}\right]^{3 / 2}+C_{1},
$$

where $0<k L \leq C \leq 1, L<1 / k$, and the constant $C_{1}=-\frac{1}{k}\left(1-C^{2 / 3}\right)^{3 / 2}$.

The tangential gravitational force acting on the mass as a function of $x$ is

$$
F_{\tau}=g \frac{y^{\prime}(x)}{\sqrt{1+\left[y^{\prime}(x)\right]^{2}}} .
$$

The normal force exerted upon the mass by the surface at the point $x$ is

$$
N=g \frac{1}{\sqrt{1+\left[y^{\prime}(x)\right]^{2}}} .
$$

\subsection{The Mass on the Dome vs. the Mass on the Pinnacle}


Having expressed the shape of the dome in the linear Cartesian coordinates, it is easy to see that not all the domes described by formula (8) would fit well for generating spontaneous motion within Newtonian mechanics.

Indeed, having fixed some "rim", $L<1 / k$, and depending on the value of the constant $C$, two distinct cases are possible.

Case 1: $C \neq 1$. Substituting $x=0$ into (7) we obtain $\left(1+\left[y^{\prime}(0)\right]^{2}\right)^{3 / 2}=\frac{1}{C}>1$, so that the first derivative of the function tends to some non-zero constant, $d$, as $x$ approaches zero. Geometrically this means that the tangent line to the dome surface at zero hits the $y$-axis at some non-zero angle - the mass arrives at the apex not exactly horizontally but at some non-zero angle. As we pass through zero into negative $x$ 's, the tangent line to the surface experiences a sudden step-like jump:

$$
\lim _{x \rightarrow 0+} y^{\prime}(x)-\lim _{x \rightarrow 0-} y^{\prime}(x)=2 d \text { for some } d \neq 0 \text {. }
$$

So $y^{\prime}(x)$ is simply not defined at $x=0$. We shall refer to this case as the mass-onthe-pinnacle scenario (Fig. 2). 


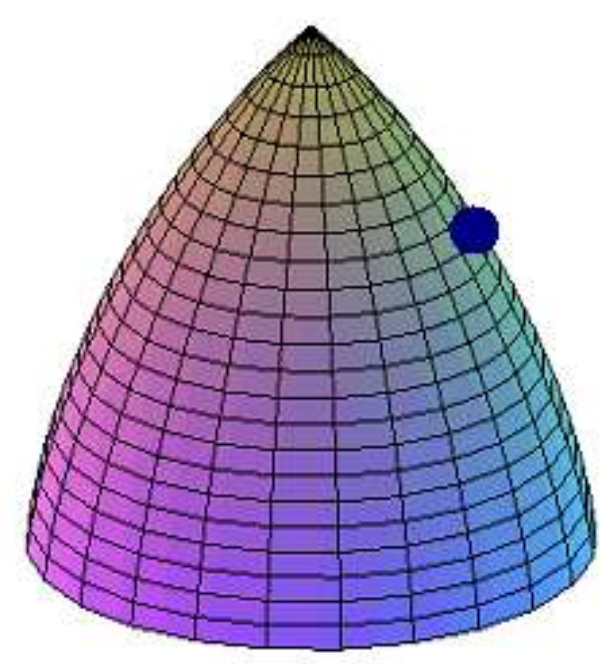

Fig. 2 The mass on the pinnacle.

Since $y^{\prime}(x)$ enters the expressions (9) and (10) for the tangential gravitational and normal forces acting on the mass, these forces appear not to be defined at zero either. As Newton's second law of motion " $F=m a$ " cannot be written for the mass at zero, the masson-the-pinnacle scenario simply does not belong in Newton's mechanics jurisdiction, and should be isolated from the discussion by an appropriate stipulation. Yet, we shall return to this case again in section 4, where it appears in regard with the Lipschitz condition.

Case 2: $C=1$. Since the constant $C_{1}=-\frac{1}{k}\left(1-C^{2 / 3}\right)^{3 / 2}$, we have $C_{1}=0$, and the expression (8) for the shape of the dome takes a relatively simple form:

$$
y(x)=-\frac{1}{k}\left[1-(1-k|x|)^{2 / 3}\right]^{3 / 2},
$$

for all $x$ out of the interval $(-L, L), L<1 / k$, for which the surface is defined.

Looking at (7), we see that the first derivative, $y^{\prime}(x)$, turns to zero as $x$ approaches zero. Geometrically this means that the mass arrives at the apex exactly horizontally. Even though the second and higher derivatives of $y(x)$ do diverge at zero, this fact by itself, at 
least directly, seems to generate no singularity or divergence in any physically meaningful parameter. We shall refer to this situation as the (proper) case of the mass on the dome (Fig. 3). All discussion that follows below will have this situation in mind.

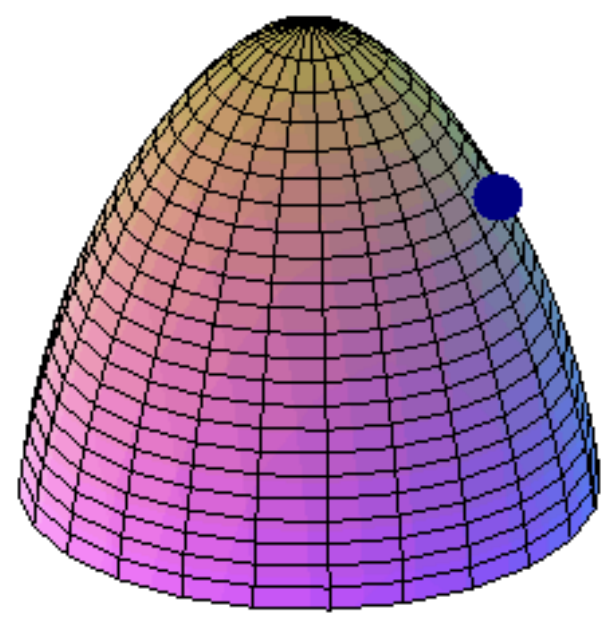

Fig. 3 The mass on the dome.

At this point we also note that in the vicinity of the apex, i.e., at the limit $|x| \rightarrow 0$, the graph behaves as a fractional power function:

$$
y(x) \approx-\sqrt{8 k / 27}|x|^{3 / 2}=-\frac{2}{3 g}|x|^{3 / 2},
$$

which, of course, should have been expected, since, for small $x$ 's, the $x$-coordinate almost coincides with the radial coordinate $r$ measured along the practically horizontal surface. We shall return to this point later in this section when trying to modify the original formulation of the problem to avoid some of its loopholes.

On the other side of the interval, as $x$ approaches the "rim", $|x| \rightarrow L$, the graph descends steeper and steeper until it hits the vertical wall at $|x|=L$. (The graph of this function appears in Fig. 1.) 


\subsection{The Mass on the Dome or the Mass in the Air?}

Further observations about the behavior of the mass on the so defined dome are in order. Consider again the three classes of trajectories produced if we give the mass at the rim some initial velocity directed at the apex along the surface: those where the mass halts before it reaches the apex and falls back to the rim; those where the mass halts exactly at the apex; and those where the mass passes the apex with some non-zero velocity and rushes over to the other side of the dome.

There is an easy and instructive way to see how things may go astray by considering the trajectories from the third class when the mass passes over the apex. As the mass proceeds through the apex with a non-zero velocity into negative $x$ 's, it continues its motion along an artillery shell like ballistic trajectory. However, in the vicinity of the apex, the dome surface descends faster than any such parabolic trajectory so that the mass necessarily detaches itself from the surface once it enters the negative $x$ 's, refusing to follow the prescribed track. (The results of numerical simulation for different velocities appear below in Fig. 4.) 


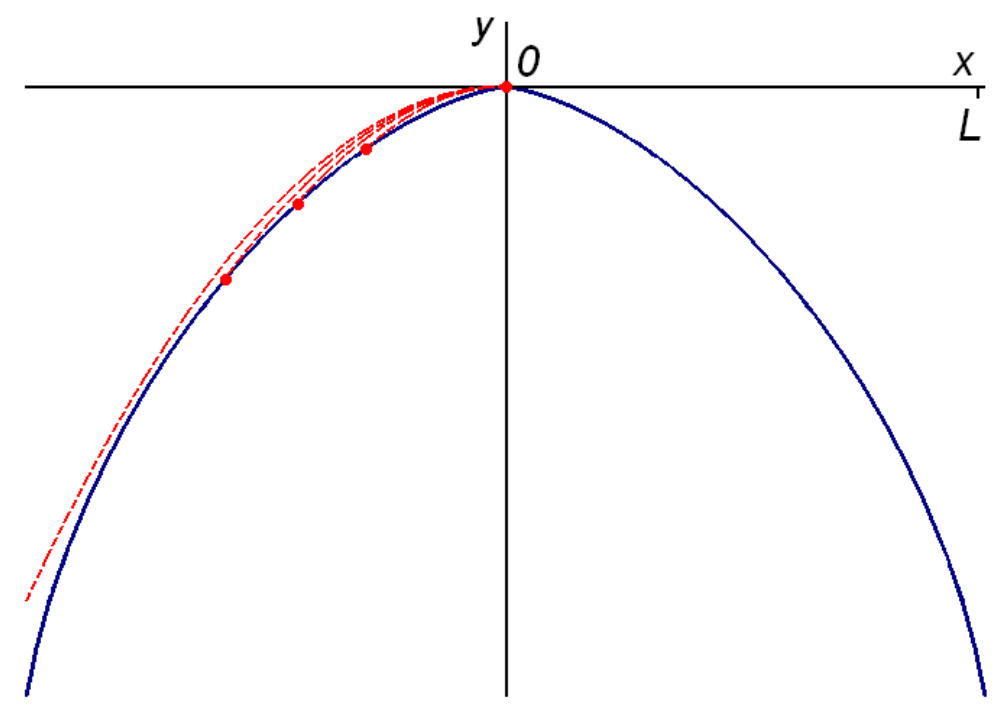

Fig. 4 Passing over the dome apex.

Similar detachment occurs in the second, more important, class of trajectories, for which the mass should halt exactly at the apex. To see this, recall that at the "rim", $x=L$, the tangential to the surface plane is exactly vertical. It means that the mass, initially positioned at the rim and given any initial velocity directed at the apex along the surface, will go up (and then fall back) precisely vertically, detaching itself from the surface and thus, again, refusing to follow its curvature. A careful analysis reveals that this is true not only for the rim $x=L$ taken as an initial position of the mass, but also for some vicinity of the rim. Indeed, for the so defined domes, there always exists a (finite) interval $\left(L_{1}, L\right)$, $0<L_{1}<L$, such that, for all initial positions of the mass (the "rims") taken within this interval, the ballistic trajectory of the mass descends slower than the dome surface immediately under it, thus causing the mass to detach from the surface. (The results of numerical simulation for different initial positions are shown below in Fig. 5.) 


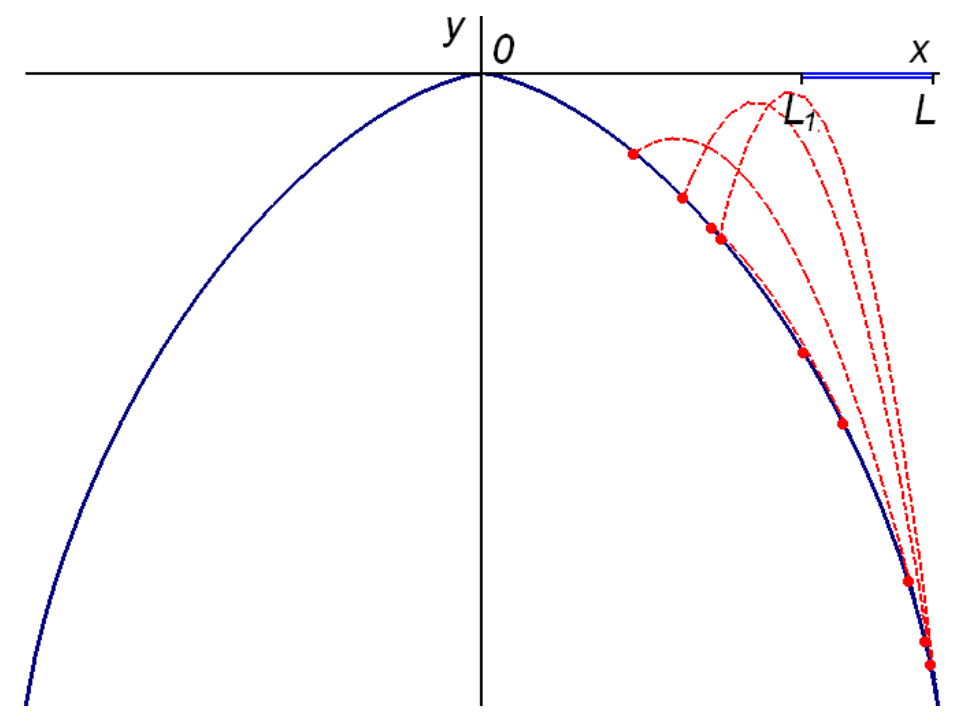

Fig. 5 Mass detachment in the vicinity of the rim.

Clearly, such initial positions would be a poor choice for being the "rim" since, as mentioned in Section 2.1, letting the mass fly along its ballistic parabolic path would irreparably ruin the time reversal argument.

Any attempt to bypass this difficulty by pushing the mass back on the prescribed track (e.g., switching to a bead-on-the-wire example) would necessarily involve additional forces (viz., the elasticity forces of the wire along which the bead would slide) without which the mass will simply refuse to follow the track. Adding new forces (external or internal), as we shall soon see, brings new and undesirable complications into Norton's original problem. ${ }^{2}$

These complications become especially important (and more subtle) when we move away from the rim to the vicinity of the apex. Unlike the previous situation, for any initial position of the mass taken within the interval $\left(0, L_{1}\right)$, the ballistic trajectory of the mass now

\footnotetext{
${ }^{2}$ This phenomenon of mass detachment is closely connected with a mechanical system's being ideal holonomic since an ideal holonomic constraint can be taken as the limiting case of a system with a large potential enegry, or, equivalently, the limiting case of an infinite force field in a neighborhood of the curve, directed toward the curve to ensure the moving point remains exactly on the curve (see Arnold 1978). I intend to expand on this point elsewhere.
} 
descends faster than the dome surface, and no detachment of the mass from the surface occurs. (The results of numerical simulation are shown in Fig. 6.)

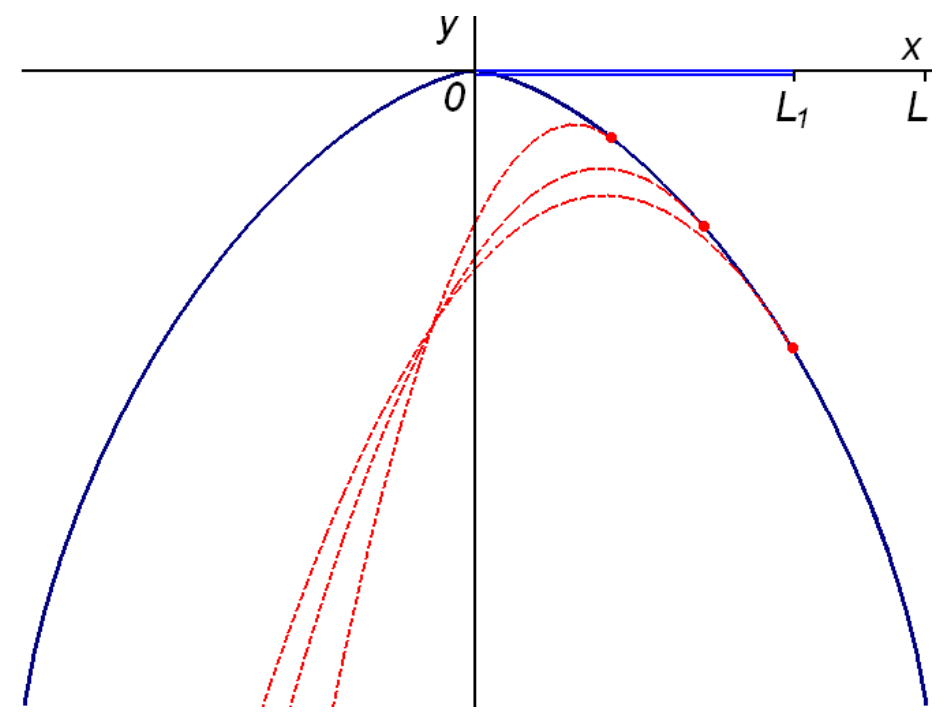

Fig. 6 No mass detachment in the vicinity of the apex.

Though we need not resort to elastic wires going through the mass to keep it from detaching itself from the surface of the dome, this is the elasticity of the dome that acquires special importance here.

At this point we can discern a general pattern that begins to emerge. We don't want to let the mass to move along its free-flight parabolic trajectory; parabolic trajectories give infinite past times for the time reversal scenarios, thus stripping the whole argument of its force. In the vicinity of the rim this can be done the invoking the elasticity forces of the wire through which the bead-mass now slides (or applying some other external force). In the vicinity of the apex these are the elasticity forces of the dome that would "straighten up" the mass path sufficiently to yield the required curvature; were it not for the elasticity forces of the dome, the mass would choose to follow its free-flight ballistic path. In any case, there is a (very strong) force field in the neighborhood of, and directed toward the surface, that 
ensures that the mass moves along the required path. Yet, as the following sections will show, no finite, however large, elasticity coefficient of the dome can allow for the time reversibility of the mass motion; only absolutely rigid dome can make the time reversal trick possible; thus the singularity in a physically meaningful parameter of the situation.

\subsection{The Mass on the Dome, Modified}

Many of the difficulties mentioned above can be overcome and will disappear if we make the following minor change in the original formulation of the problem. Namely, instead of defining the surface by $y(r)=-\frac{2}{3 g} r^{3 / 2}$, in curvilinear terms of the distance $r$ traveled by the mass along the surface, we define it, just as (12) suggests, in the usual linear Cartesian coordinates by

$$
y(x)=-\frac{2}{3 g}|x|^{3 / 2} .
$$

This way, first, the situation no longer harbors the mass-on-the-pinnacle case in which the slope of the surface experiences a sudden step-like jump at the apex. The mass moving along the surface will now always arrive at zero exactly horizontally and no qualifications to the contrary are necessary.

Second, the surface is now defined for all $x^{\prime} \mathrm{s}$, not just for all $x$ out of some interval $(-L, L), L<1 / k ;$ no more "preferred" "rims" or vertical walls.

Third, with any arbitrary ( $x$-positive) point on the dome taken as the motion starting point (the "rim"), no detachment of the mass from the surface when it starts at its "rim" ever occurs; no need to resort to elastic wires to keep the mass on the prescribed track. 
This does not, however, prevent the mass to detach from the surface for the third class of trajectories once the mass passes the apex with non-zero velocity as in Fig. 4. Fortunately, these trajectories play no role in Norton's time reversal argument, so we shall simply let the mass disappear from our attention once it vanishes behind the apex into the other side.

As the following sections will show, the so modified mass on the dome example inherits all the strangeness of being a source of spontaneous motion generation with no need to deal with the above discussed loopholes and unnecessary qualifications.

\section{The Lipschitz Condition}

We recall that the function $x(t)$ satisfying the initial condition $\left(t_{0}, x_{0}\right)$ is a solution of the differential equation determined by a vector field $v$

$$
\dot{x}=v(x)
$$

if the following identity holds for all $t$ in the interval $I$ on which $x(t)$ is defined:

$$
\frac{d x}{d t}=v(x(t)) \text {, and } x\left(t_{0}\right)=x_{0} .
$$

Every differential equation (13) defines a direction field of this equation in the plane:

the line attached at the point $(t, x)$ has slope $v(x)$. If $x_{0}$ is a singular point of the vector field, i.e., $v\left(x_{0}\right)=0$, then $x(t)=x_{0}$ is a solution of the equation (13) satisfying the initial condition $x\left(t_{0}\right)=x_{0}$. Such a solution is called an equilibrium position or stationary solution. 
Let $v(x)=\operatorname{sgn}(x)|x|^{3 / 4}$. For such a field, (13) has more than one solution, e.g., the solutions $x_{1}(t)=0$ and $x_{2}(t)=|t / 4|^{4}$ satisfy the same initial condition $(0,0)$. In fact, (13) has a whole 1-parameter family of solutions obtained by gluing together the corresponding halves of the two solutions, $x_{1}(t)=0$ and $x_{2}(t)=[(t-T) / 4]^{4}$, at some arbitrary time $T>0$. (This situation is typical in that if (13) has more than one solution, then it has a "continuum" (i.e., a closed connected set) of solutions.) In the general theory of ordinary differential equations it is hardly a surprising fact; if the direction field $v$ is continuous but nondifferentiable (only Hölder continuous), the solution with initial condition in the equilibrium position may fail to be unique. Indeed, for any $v(x)=\operatorname{sgn}(x)|x|^{\alpha}$, where $0<\alpha<1$, there always exists a family of branching solutions for (13) that satisfy the same initial condition $(0,0)$ (Fig. 7):

$$
x(t)= \pm\left[(1-\alpha)(t-T)_{+}\right]^{1 /(1-\alpha)}
$$

where $f_{+}=\max (f, 0)$, for an arbitrary $T$. 


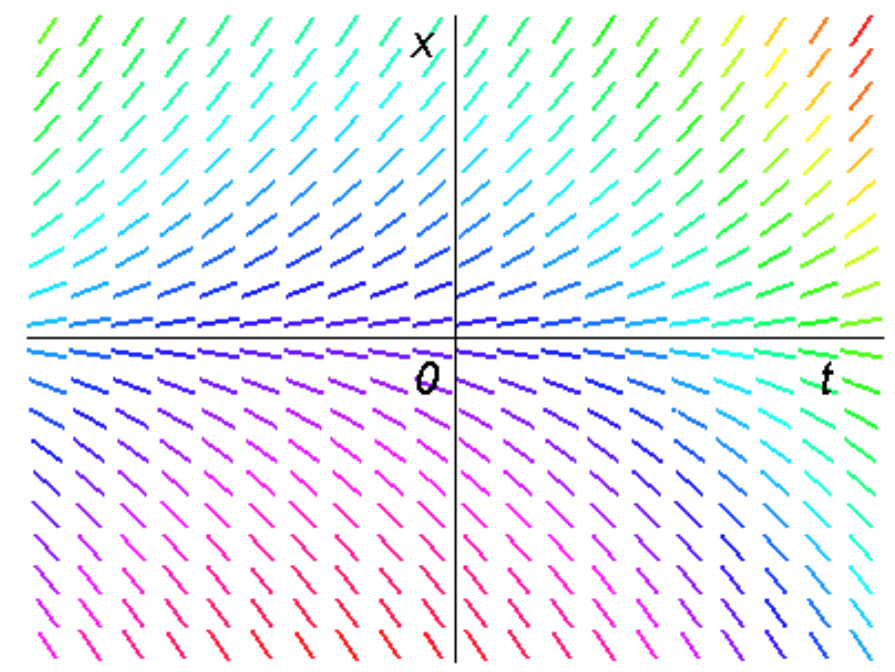

Fig. 7 The direction field for $\alpha=3 / 4$.

Geometrically, the reason for non-uniqueness in these cases is that the velocity decreases too slowly when approaching the equilibrium position. As a result the solution manages to reach the singular point in a finite time. It turns out that the smoothness of $v$ guarantees the uniqueness in these cases. This observation needs more elaboration.

Let us assume that $x(t)$ is a solution of the equation $\dot{x}=v(x)$ with a smooth righthand side $v$. We shall suppose that $x\left(t_{0}\right)=x_{0}$ is an equilibrium position and $x\left(t_{1}\right)=x_{1}$ is not (Fig. 8). 


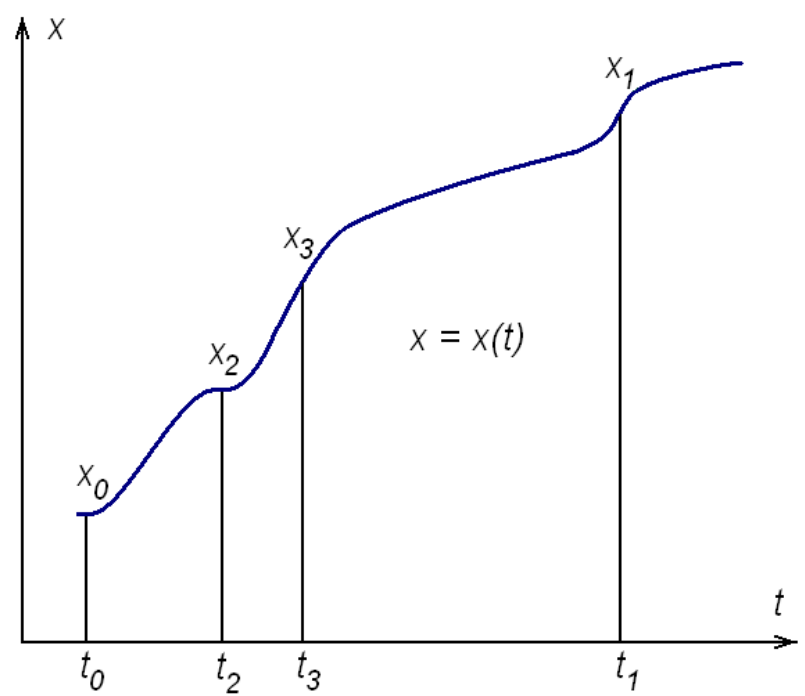

Fig. 8

On the interval between $t_{0}$ and $t_{1}$ consider the instant $t_{2}$ closest to $t_{1}$ such that $v\left(x\left(t_{2}\right)\right)=0$. By Barrow's formula for any point $t_{3}$ between $t_{2}$ and $t_{1}$ we have

$$
t_{3}-t_{1}=\int_{x_{1}}^{x_{3}} \frac{d \xi}{v(\xi)}, x_{3}=x\left(t_{3}\right)
$$

If the function $v$ is smooth, then the integral tends to infinity as $x_{3}$ tends to $x_{2}$. Indeed, the slope of the chord of the graph of a smooth function on an interval is bounded (Fig. 9), so that $|v(\xi)| \leq k\left|\xi-x_{2}\right|$, where the constant $k$ is independent of the point $\xi$ of the interval $\left[x_{1}, x_{2}\right]$. 


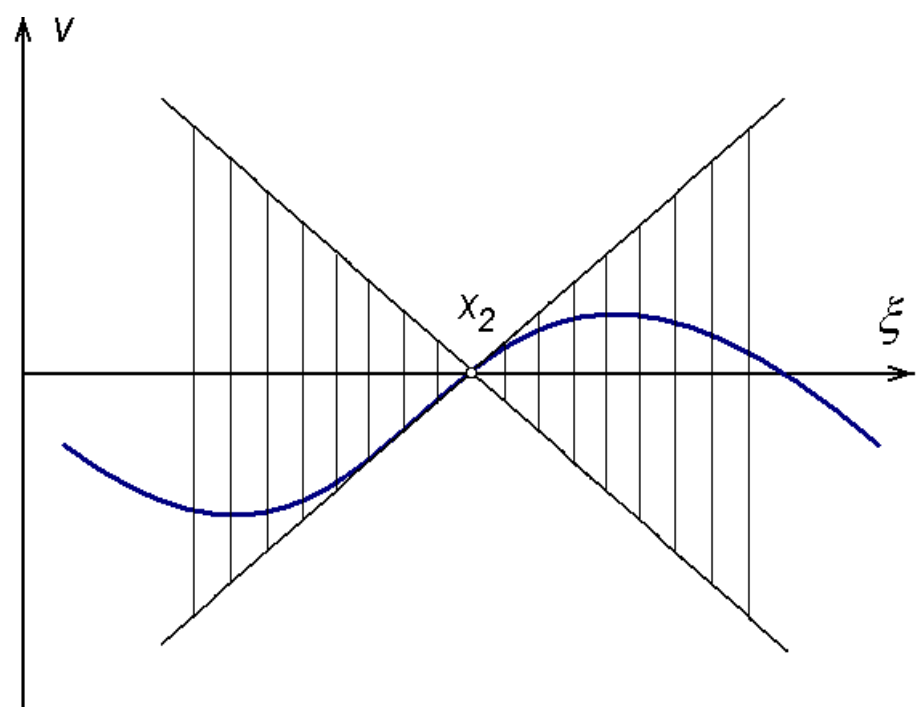

Fig. 9

Thus

$$
\left|t_{3}-t_{1}\right| \geq\left|\int_{x_{1}}^{x_{3}} \frac{d \xi}{k\left(\xi-x_{2}\right)}\right| .
$$

The latter integral is easily calculated; it tends to infinity as $x_{3}$ tends to $x_{2}$. It is easy to verify this without even calculating the integral: it must be equal to the time of transit between the two points in the linear field, and this time tends to infinity when one of the points tends to the equilibrium position.

Thus the number $\left|t_{2}-t_{1}\right|$ is larger than any preassigned number. So the solution with initial condition in an equilibrium position cannot assume values that are not equilibrium positions. Therefore if $x\left(t_{0}\right)$ is an equilibrium position, we have $v(x(t)) \equiv 0$ for all $t$. Consequently $\dot{x}(t) \equiv 0$, i.e., $x(t)$ is a constant. The uniqueness is now proved. ${ }^{3}$

Note that the main point of the proof was the comparison of a motion in a smooth field with a more rapid motion in a suitable linear field (i.e., parabolic trajectories). For the

\footnotetext{
${ }^{3}$ The proof is due to Arnold (1992).
} 
latter motion the time to enter an equilibrium position is infinite, and consequently it is $a$ fortiori infinite for the slower motion in the original field. Indeed, it can be shown that a sufficient condition for uniqueness of the solution with initial value $x_{0}$ is that the integral $\int_{x_{0}}^{x} \frac{d \xi}{v(\xi)}$ diverge at $x_{0}$

The condition that $|v(\xi)| \leq k\left|\xi-x_{2}\right|$, where the constant $k$ is independent of the point $\xi$ of the interval $\left[x_{1}, x_{2}\right]$ (i.e., the condition that the slope of the chord of the graph be bounded), is called a Lipschitz condition and the constant $k$ a Lipschitz constant. ${ }^{4}$ It can be shown that a sufficient condition for uniqueness is that the right-hand side function $v$ satisfy a Lipschitz condition $|v(x)-v(y)| \leq k|x-y|$ for all $x$ and $y$.

It is by no accident that we chose the function $|v|=|x|^{3 / 4}$ to exemplify the Lipschitz discontinuity. Writing down the energy conservation relation for a unit mass sliding on the surface of the axially symmetric dome defined by the equation $y(x)=-|x|^{3 / 2}$ gives us

$$
\frac{\dot{x}^{2}}{2}=g|y(x)|,
$$

so that

$$
|\dot{x}|=\sqrt{2 g}|y(x)|^{1 / 2}=\sqrt{2 g}|x|^{3 / 4}
$$

\footnotetext{
${ }^{4}$ More generally, if $|f(\xi)-f(x)| \leq k|\xi-x|^{\beta}$ for given $x$ and all $|\xi-x|<\delta$, where $k, \beta$ are independent of $\xi$, and $\beta>0$, and $\alpha$ is the upper bound of all the $\beta$ such that a finite $k$ exists, $f(\xi)$ is said to satisfy a Lipschitz condition of order $\alpha$ at $\xi=x$. If a Lipschitz condition of order $\alpha$ is satisfied at $x$, it can be shown that $f^{\prime}(x)=0$. If at every point of the interval it is satisfied for some $\alpha>1$, then $f^{\prime}(x)=0$ throughout the interval. Hence $f(x)$ is constant; consequently only the case $0<\alpha \leq 1$ is of much interest.
} 
which, to the factor of $\sqrt{2 g}$, coincides with our $|v|$. The right-hand side of the expression

is non-Lipschitz: its derivative, $\frac{3 \sqrt{2 g}}{4}|x|^{-1 / 4}$, is unbounded in the neighborhood of the origin. As a result, the uniqueness theorem does not apply. Gluing together in a smooth manner the corresponding halves of the two solutions, $x_{1}(t)=0$ and $x_{2}(t)=[(t-T) / 4]^{4}$, at some arbitrary (positive) time $T$ reproduces Norton's anomalous solutions. Thus the existence of anomalous non-trivial solutions in Norton's case can be wholly attributed to (spatial) Lipschitz-discontinuity of the potential well wherein the mass moves. We shall call such solutions (spatial) Lipschitz indeterministic.

A further observation clarifying the role of the Lipschitz condition is in place. A Lipschitz condition is weaker than that for the function $v$ to be smooth. Indeed, the uniqueness theorem holds in the case when the first derivative of $v$ exists but is discontinuous. What that means with respect to our situation is that the mass-on-thepinnacle case cannot be amended by defining the otherwise undefined values of the derivative $y^{\prime}(x)$. Try, for instance, to define it as the right-hand limit of the derivative at zero (i.e., regard the mass at apex positioned as if it is still on the right-hand side of the surface only):

$$
y^{\prime}(0):=\lim _{x \rightarrow 0+} y^{\prime}(x)<0 .
$$

Now that the derivative exists but is discontinuous, the uniqueness theorem comes into play, and only one solution survives. Which one and why? Substituting this value of $y^{\prime}(0)$ into (9) we see that, at the apex, there is always a non-zero tangential gravitational force $F_{\tau}$ pushing the mass downward. It is to say that, after having reached the apex and 
having momentarily halted, the mass will necessarily turn back and fall down to where it came from. The situation becomes no more paradoxical than throwing a stone vertically in the air, seeing it halt after some finite time, and then catching it back again. The trivial solution $x_{1}(t)=0$ is impossible on so defined pinnacle surface exactly for the same reasons that make it impossible to have the stone hang in the midair forever once it has reached its maximal altitude. (A similar argument applies if one tries to define $y^{\prime}(0)$ as the left-hand limit of the derivative at zero.)

Defining the derivative by zero, instead, leaves only the trivial solution. Once the mass is perched on the "flat" top of the dome, it will never move away. Any slightest shift in its position will lead to a sudden jump in both magnitude and directionality of the tangential and normal forces acting on the mass. No non-trivial solutions of the equation can accommodate these jumps. Causality reigns.

\section{Asymptotic Reasoning and Time Irreversibility}

\subsection{Models and Idealizations}

Scientific theories using mathematical models "approximate" or "idealize" in one way or another. Whereas much attention in philosophy of science has been drawn to the role of idealization in the development of scientific theories (e.g., Cushing (1990)), it is the applicability of the theories or models that shall be of main interest in this section. More specifically, we shall focus on the role that certain idealizations play in Norton's stochastic motion generation.

In his recent book (2002) Robert Batterman discusses what he calls asymptotic reasoning in physics, i.e., the qualitative analysis of behavior of physical theories in the 
neighborhood of singular limits, and its relevance to philosophical issues of explanation, reduction and emergence. Batterman argues that many physically and philosophically important theories and models involve a new and powerful category of explanation based on asymptotic reasoning that has been totally overlooked by philosophers of science.

Whereas much of Batterman's efforts have been directed on issues of intertheoretical reduction and the development of the new type of explanation with the idea of emergence, we shall be applying the (infinite) asymptotic reasoning to see whether a particular model, namely, Norton's mass-on-the-dome example, involves any physically inadmissible idealizations.

One of the key ideas involved in infinite asymptotic reasoning is a commonplace fact of mathematics that finite and infinite compositions may differ in their essential properties. (Thus, a finite intersection of open sets is always an open set, but an infinite intersection of open sets can yield a closed set). If some property is preserved at any finite stage of a finite sequence of operations, there is no guarantee that this property will be preserved in the transition to the infinite stage. One may argue that many paradoxes in philosophy can be traced down to exactly this illegitimate projection - incorrectly projecting properties from finite to infinite compositions. ${ }^{5}$ This can be seen as a particular application of a more general pattern encountered routinely in such mathematical disciplines as the system stability and control theory, the theory of parameter sensitivity in dynamical systems, catastrophe theory, and robotics.

Unlike the more traditional approaches in which to determine the properties of a system has been to exhibit a complete set of exact solutions of the equations describing this system, and then to study the properties of these solutions, in catastrophe theory it is

\footnotetext{
${ }^{5}$ See, e.g., Earman and Norton (1999) for applying this reasoning to argue against known supertask
} paradoxes. 
realized that in many instances it is only information of a qualitative nature, or only limited quantitative information, which is the ultimate goal of the study of some systems of equations. In such cases a full spectrum of solutions to an equation, obtained by much hard work (if at all), may be a hindrance rather than a help in understanding the qualitative properties of the equation or system of equations (Gilmore 1981).

As a part of mathematics, catastrophe theory is a theory about singularities. Many interesting phenomena in nature (or their mathematical models) involve some discontinuities - breaking of a wave, the division of a cell or the collapse of a bridge. When applied to scientific theories, it deals with the properties of discontinuities directly, without reference to any specific underlying mechanism. This makes it especially appropriate for the study of systems whose inner workings are not known, or too complicated, and for situations in which the only reliable observations are of discontinuities.

\subsection{Stability and Parameter Sensitivity}

In robotics, to maintain system stability has been the prime concern when designing any practical machine. There always exists a certain discrepancy between an actual (realoperating) and the nominal (theoretical) trajectories of any system. This discrepancy is partly due to various inherently approximational schemes in system identification, and partly due to possible further parameter variations stimulated by environmental changes. Thus, special attention should be paid to the evaluation of possible system parameter variations, and their effects on system's functional performance or "output". 6

To illustrate the concepts of stability and parameter sensitivity let us consider a familiar classical system - a pendulum. A simple gravity pendulum - a weight on the end of

\footnotetext{
${ }^{6}$ See, e.g., Eshlami (1991).
} 
a rigid rod, which, when given an initial push, swings back and forth under the influence of gravity over its central (lowest) point. As is known, the oscillations (not necessarily small) of the "ideal" pendulum are described by the following system of differential equations:

$$
\dot{x}_{1}=x_{2}, \dot{x}_{2}=-\omega^{2} \sin x_{1}, \omega=\sqrt{l / g} \text {, }
$$

where $x_{1}$ is the angle of deviation from the vertical, $x_{2}$ is the angular velocity, $l$ is the length of the pendulum, and $g$ is the acceleration due to gravity.

The corresponding vector field in the phase plane with coordinates $x_{1}, x_{2}$ is just

$$
v_{1}=x_{2}, v_{2}=-\omega^{2} \sin x_{1},
$$

with singular points $x_{1}=m \pi, x_{2}=0$ (Fig. 10).

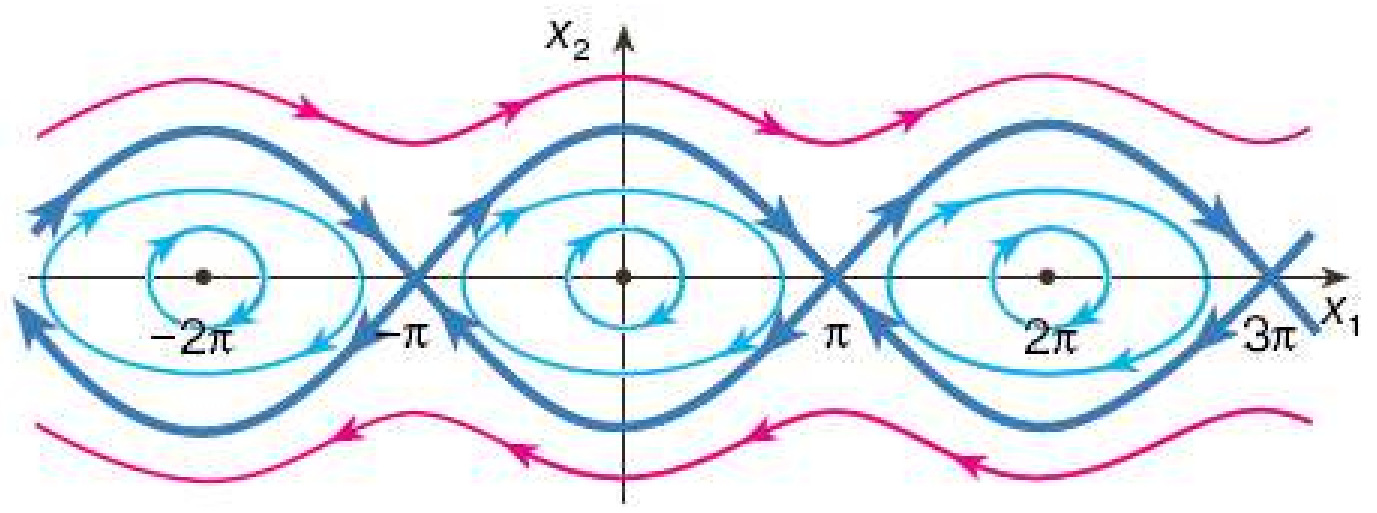

Fig. 10 Phase-space of a simple gravity pendulum

If we restrict the motion of the pendulum to a relatively small amplitude, i.e., $\left|x_{1}\right|<<1$, the solution is a well-known harmonic oscillatory function:

$$
x_{1}(t)=x_{0} \cos (t / \omega), \quad x_{2}(t)=-(1 / \omega) x_{0} \sin (t / \omega)
$$

where $x_{0}$ is the largest angle attained by the pendulum.

Period of the small oscillations is 


$$
T_{0}=2 \pi / \omega
$$

For amplitudes beyond the small angle approximation, the exact period cannot be evaluated in terms of elementary functions and can only be written in the form of the elliptic function of the first kind:

$$
T=\frac{4}{\omega} E\left(\frac{\sin x_{0}}{2}, \frac{\pi}{2}\right)
$$

where $E(\psi, \varphi)$ is Legendre's elliptic function of the first kind:

$$
E(\psi, \varphi)=\int_{0}^{\varphi} \frac{1}{\sqrt{1-\psi^{2} \sin ^{2} \xi}} d \xi .
$$

The value of the elliptic function can also be computed numerically by using the following series:

$$
T=T_{0}\left[1+\left(\frac{1}{2}\right)^{2} \sin ^{2}\left(\frac{x_{0}}{2}\right)+\left(\frac{1 \cdot 3}{2 \cdot 4}\right)^{2} \sin ^{4}\left(\frac{x_{0}}{2}\right)+\left(\frac{1 \cdot 3 \cdot 5}{2 \cdot 4 \cdot 6}\right)^{2} \sin ^{6}\left(\frac{x_{0}}{2}\right)+\ldots\right]
$$

A number of assumptions are built in this model: the bob of the pendulum is a pointmass; the rod on which the bob is swinging is massless and absolutely rigid; motion occurs in a vertical plane; there is no air resistance and friction at the nail, the material of which the bob is made is irrelevant to the study of the question, acceleration due to gravity does not depend on the position of the bob; there is no gravitational influence of the nearby objects at the mass, etc. Taken seriously, many of these idealizations are plainly unphysical (or physically inadmissible) in that they can never be achieved in practice for principle reasons, but, of course, no one is tempted to think that this "unphysicality" is indispensable to the relevant theory or that the theory would be absolutely unworkable without them.

A typical way how such conceptual "frauds" are dealt away in the teaching of science can be illustrated by the following excerpt: 
One of the fundamental concepts of mechanics is that of a [material point]. By this we mean a body whose dimensions may be neglected in describing its motion. The possibility of so doing depends, of course, on the conditions of the problem concerned. For example, the planets may be regarded as [material points] in considering their motion about the Sun, but not in considering their motion about their axes. (Landau and Lifschitz 1976, p. 1)

Implicit in such stipulations are our intuitions about the stability (or robustness) of the behavior of the system with respect to disturbances or changes made to various parameters of the system. That is this feature of a system to change its behavior insignificantly when the various parameters of the system are changed insignificantly that legitimizes some physical features to be idealized, or "neglected", as in the example above. This point needs more elaboration.

We recall that an equilibrium point $\boldsymbol{x}_{0}$ of a system of differential equations,

$$
\dot{\boldsymbol{x}}=\boldsymbol{v}(\boldsymbol{x}(t)),
$$

is locally Lyapunov stable at $t=t_{0}$ if all solutions of this equation which start near $\boldsymbol{x}_{0}$ (i.e., with their initial conditions in a neighborhood of $\boldsymbol{x}_{0}$ ) remain near $\boldsymbol{x}_{0}$ for all time, i.e., if for any $\varepsilon>0$ there exists a $\delta\left(\varepsilon, t_{0}\right)>0$ such that

$$
\text { if }\left\|\boldsymbol{x}\left(t_{0}\right)-\boldsymbol{x}_{0}\right\|<\delta \text { then }\left\|\boldsymbol{x}(t)-\boldsymbol{x}_{0}\right\|<\varepsilon \text {, for all } t \geq t_{0}
$$

for some appropriate choice of the norm $\|\ldots\|$.

The equilibrium point $\boldsymbol{x}_{0}$ is said to be locally asymptotically stable if $\boldsymbol{x}_{0}$ is locally stable (in the sense of Lyapunov) and, furthermore, all solutions starting near $\boldsymbol{x}_{0}$ tend 
towards $\boldsymbol{x}_{0}$ as $t \rightarrow \infty$. Thus, the pendulum has a locally stable equilibrium point (but not asymptotically stable) when the pendulum is hanging straight down and an unstable equilibrium point when it is pointing straight up. (If the pendulum is damped, the stable equilibrium point is locally asymptotically stable.)

Let us frame this situation differently in the following terms. Instead of disturbing the initial conditions of the system, we can talk of changing (smoothly) the total system energy taken as a parameter, and observe the qualitative changes in the behavior of the system. As we start with small system energies (i.e., the initial conditions are around the point $(0,0)$ in the phase-space diagram), the trajectories of the pendulum bob are closed curves; the pendulum performs back-and-forth oscillations. Increasing, in a smooth manner, the total energy of the system, we will eventually reach a point when, suddenly, the pendulum bob ceases to track a closed curve in the phase-space; in fact, it ceases to move at all once it takes an (unstable) vertical position with the zero velocity, and remains in this unmovable state ever since. This position is unstable - any, however light, disturbance of the pendulum returns it to the back-and-forth oscillatory motion.

If we increase the total energy even more, the pendulum starts a rotating motion, corresponding to non-closed curves in the phase-space diagram, resembling more and more straight lines as we push the energy still up. Thus, depending on the (non-zero) value of the chosen parameter (system's total energy), there exist three distinct behaviors of the system that of a back-and-forth oscillations (closed phase trajectories), that of a halted (though unstable) position (a phase trajectory is just a single point), and that of a rotational motion about the axe of the pendulum (non-closed phase trajectories).

Depending on a particular problem in question, any other parameter of the system may be chosen to be disturbed or manipulated. An interesting (and important to us) case is 
when we choose to manipulate the rod's elasticity coefficient $k$, and see whether the behavior of the system will be, in some appropriate sense, robust under these perturbations.

Suppose, for example, that we are given a system of differential equations describing the behavior of a gravitational pendulum, in which the elasticity of the rod is not assumed infinite but appears explicitly. Suppose further that, in this system of equations, the elasticity coefficient $k$ of the rod is replaced by a new coefficient $k^{\prime}=k+\delta k, \boldsymbol{x}(t)$ is a solution of the original equation, and $\boldsymbol{x}^{\prime}(t)$ is a solution of the equation with the changed $k^{\prime}$ . For $\boldsymbol{x}(t)$ to be a robust solution, we could require that for any appropriate disturbances $\delta k$ there exists a $\delta\left(\varepsilon, t_{0}\right)>0$ such that

$$
\left\|\boldsymbol{x}^{\prime}(t)-\boldsymbol{x}(t)\right\|<\delta \text {, for all } t \geq t_{0} .
$$

This is the latter sense of robustness that is essential for the possibility of the "unphysical" models' being used in simulating the behavior of physical systems. Thus, by a pendulum with infinitely rigid rod we could now understand a series of (physically legitimate) approximations to the original problem, with finite but arbitrarily large and ever increasing elasticity coefficients, $k^{\prime} \mathrm{s}$, as long as (1) the series converges (in some appropriate sense) to some limiting solution (e.g., if the modulus of any two consecutive solutions can be made arbitrarily small by further increasing the corresponding elasticity coefficients), and (2) this limiting solution has the same essential properties that any finitely approximate solution has.

\subsection{The Mass on the Dome and Time Irreversibility}

What does it all have to do with the mass-on-the-dome example? As we have seen earlier, the generation of non-uniqueness in this situation is due to the violation of the (spatial) 
Lipschitz condition. The violation occurs at one point of the dome only - the apex. If we grant infinite divisibility of matter and neglect the issues with the vanishing Lebesgue measures of the initial conditions, a more philosophically interesting issue arises, namely, that of time irreversibility of the solutions.

Note that, even though the Lipschitz condition is violated at a single point, the apex, this violation depends on the behavior of the dome at the infinitesimal vicinity of the apex; it is crucial that the dome shape's first derivative experiences unbounded growth in the infinitesimal proximity of the apex. An arbitrarily small vicinity of the apex has been chosen, the particular shape of the path that the mass moves along outside of this vicinity is absolutely immaterial to the spontaneous motion at the apex to begin; no points of the dome outside of this area in any way affect the spontaneous generation of motion at the apex.

Now, we know that in a (real) body that is not deformed, the arrangement of the molecules correspond to a state of thermal equilibrium; all parts of the body are in mechanical equilibrium. This means that, if some portion of the body is considered, the resultant of the forces on that portion is zero. When a deformation occurs, the arrangement of the molecules is changed, and the body ceases to be in its original state of equilibrium. Forces therefore arise which tend to return the body to equilibrium. These internal forces which occur when a body is deformed are called internal stresses. If no deformation occurs, there are no internal stresses.

The internal stresses in a real physical body are due to molecular forces, i.e., the forces of (electro-magnetic) interaction between the molecules. An important fact in the theory of elasticity is that the molecular forces have a very short range of action. Their effect extends only to the neighborhood of the molecule exerting them, over a distance of the same order as that between the molecules, whereas in the theory of elasticity, which is a 
macroscopic theory, the only distances considered are those large compared with the distances between the molecules. The range of action of the molecular forces should therefore be taken as zero in the theory of elasticity. We can say that the forces which cause the internal stresses are, as regards the theory of elasticity, "near-action" forces, which act from any point only to neighboring points. Hence it follows that the forces exerted on any part of the body by surrounding parts act only on the surface of that part (Landau and Lifshitz, 1959). Another consequence of such treatment of infinitely divisible matter is that no internal stresses in the theory of elasticity can be non-smooth or have divergent or singular derivatives.

Coming back to the mass-on-the-dome example, the trajectories of the first class (where a mass is given an initial push up and it stops exactly at the apex) can now be shown robust under the ever increasing rigidity of the dome in the following sense.

Take any arbitrarily large elasticity coefficient $k_{1}$ of the dome. Place a mass on the "rim" (any fixed point on the dome other than the apex), give it a push up so that the mass halts exactly at the apex. Write down the formal exact solution of such a motion (non-trivial at all, given that we are not to neglect any dome deformation effects). Call this solution, as a function of time, $\boldsymbol{x}_{1}(t)$. Double (triple, or whatever) the elasticity coefficient $k_{1}$ to get a new $k_{2}$. Get a similar solution $\boldsymbol{x}_{2}(t)$. Proceed in the similar manner to get an infinite series of solutions: $\boldsymbol{x}_{1}(t), \boldsymbol{x}_{2}(t), \ldots, \boldsymbol{x}_{i}(t), \ldots$. It can be shown that, in some appropriate sense, this sequence converges to some limiting solution, $\boldsymbol{x}(t)$, which we would call a solution for an absolutely rigid dome (the elasticity coefficient is infinite). 
Notice now what happens if we try to time-reverse the situation. Fix some finite $k_{1}$, place a (point) mass on the top of the dome, and ... Will the mass move down? Given that the mass is a point mass (to hit exactly the apex) and the internal stresses in the (nonabsolutely rigid) dome are smooth, there exists no way the surface of the dome hits the apex in a non-smooth manner. No Lipschitz-discontinuity - no non-uniqueness - the mass will stay on top forever! Try doubling, tripling, etc., the elasticity of the dome - you get the same picture. As long as the dome elasticity stays finite (and no matter how big), there is no spontaneous motion generation when the mass is on the top!

The situation changes essentially if we take an absolutely rigid dome with no deformations at all; only then, given the nature of the internal forces that we have, could the Lipschitz condition be violated and the mass slide down. Thus, a limiting solution of this family of solutions exhibits a property (of being non-unique), whereas any solution in an approaching series (corresponding to a physically plausible situation) fails to exhibit this property. The only way to generate non-Lipschitz spontaneous motion of the mass down the dome is to have an absolutely rigid dome; any diversion from actual infinity in the elasticity coefficient would result in the mass staying on top forever.

There is an important lesson to be learned from this example to be very careful in using time-reversibility tricks. As Norton's mass on the dome shows, these are the cases the real metaphysical power of which may essentially depend on the nature of idealizations done in those models, and the techniques of asymptotic reasoning may prove crucial in elucidating these issues.

\section{Non-Lipschitz Indeterministic Solutions and the Markov Condition}




\subsection{Generalized Flows in Fluid Dynamics}

In this section we draw the results from fluid dynamics to further support the view that the Lipschitz condition should be taken is an important implicit assumption within Newtonian mechanics and that the failure to recognize it as such may unsurprisingly lead to physically impossible situations.

Consider the following transport equation for the scalar field $\theta(x, t)$ in $(x, t) \in \mathbb{R}^{d} \times[0, \infty):$

$$
\frac{\partial \theta}{\partial t}+(v(x, t) \cdot \nabla) \theta=0,\left.\quad \theta\right|_{t=o}=\theta_{0}
$$

In the classical theory of partial differential equations it is known that if the velocity field $v \in \mathbb{R}^{d}$ is bounded and continuous in $(x, t)$ and Lipschitz continuous in $x$, then (17) can be solved uniquely by the method of characteristics. Denote by $\varphi_{s, t}(x)$ the solution of

$$
\frac{d x}{d t}=v(x, t)
$$

starting at $x$ at time $s$, i.e., with the initial condition $x(s)=x$. The solution of (17) is then given by the following expression:

$$
\theta(x, t)=\theta_{0}\left(\varphi_{0, t}^{-1}(x)\right)=\theta_{0}\left(\varphi_{t, 0}(x)\right)
$$

The map $\varphi_{s, t}: \mathbb{R}^{d} \mapsto \mathbb{R}^{d}$ satisfying the following four properties:

(a)

(b) $\quad \varphi_{s, t}(x)$ is continuous in $s, t, x$;

(c) $\quad \varphi_{\tau, t}\left(\varphi_{s, \tau}(x)\right)=\varphi_{s, t}(x)$ for all $s, t, \tau$ and all $x$;

(d) $\quad \varphi_{s, t}(x): \mathbb{R}^{d} \mapsto \mathbb{R}^{d}$ is a homeomorphism for all $s, t$ 
is called a flow of homeomorphism or, in short, a flow. ${ }^{7}$

These classical results are inapplicable when $v$, though bounded and continuous in $(x, t)$, fails to be Lipschitz continuous in $x$. In such cases no standard flow satisfying (a)-(d) can be associated with the ODE in (18) since the solution of this equation may fail to be unique. Since the solutions of (18) typically branch (i.e., (18) have more than one solution for the same initial condition), no forward-in-time map can be associated with such solutions. Similarly, no backward-in-time map can be associated with the solutions of (18) because they may coalesce on each other in finite time. This situation is unfortunate since transport in non-Lipschitz velocity fields may be physically motivated, e.g., for the problem of turbulence. $^{8}$

Formally, the standard way to deal with this situation in general case is to randomize the set of maps which can be associated with the solutions in (18) by selection at the branching points thus defining a random field. Then a generalized flow associated with (18) can be defined as a random field with parameter $(s, t, x)$ constructed by assigning a probability measure on the set of all maps associated with the solutions of (18). ${ }^{9}$ The generalized flows obtained in this way are typically non-degenerate random fields, i.e., the measure is not concentrated on a single point, due to branching.

As far as the modeling of the underlying physical processes is concerned, to pick the probability measure and single out physically relevant generalized flows the following regularization procedure is used. Instead of the original problems described by (17) and (18), the regularized problems with unique solutions are considered and the generalized

\footnotetext{
${ }^{7}$ Exposition is due to W. E and Vanden-Eijnden (2003).

${ }^{8}$ The classical theory of Kolmogorov (1941) predicts that the solution of the Navier-Stokes equation in three dimensions in only Hölder with exponent $1 / 3$ in the limit of zero viscosity.

${ }^{9}$ For a more rigorous definition of generalized flows and their properties see Appendix A, W. E and VandenEijnden (2003).
} 
flows are obtained as limits of the standard (stochastic) flows associated with these regularized problems.

Consider first the regularization by smoothing of the velocity around the points of Lipschitz discontinuity (the $\varepsilon$-limit process). Here the original equation (17) is understood as the limiting equation for the following motivating (and physically legitimate) problem:

$$
\frac{\partial \theta}{\partial t}+\left(v^{\varepsilon}(x, t) \cdot \nabla\right) \theta=k \Delta \theta,\left.\quad \theta\right|_{t=o}=\theta_{0},
$$

where $k$ is the molecular diffusivity and $v^{\varepsilon}$ is a mollified version of $v$ on the scales $|x| \ll \varepsilon$ (e.g., if $v$ solves Navier-Stokes equation, $\varepsilon$ is the characteristic length scale associated with the kinematic viscosity). Unlike the original transport equation, (20) has a unique solution if either $k$ or $\varepsilon$ are positive. The generalized flow is then taken as the limit as $\varepsilon \rightarrow 0$ of the stochastic flow associated with (20), provided this limit can be defined in a suitable way.

Secondly, some Brownian motion (the $k$-limit process) can added to the dynamics in (18) to obtain a unique (stochastic) flow associated with the solutions of

$$
d x=v^{\varepsilon}(x, t) d t+\sqrt{2 k} d \beta(t)
$$

where $\beta(\cdot)$ is a $d$-dimensional Wiener process (Stroock and Varadhan, 1969, 1979). The fact that the term $\sqrt{2 k} d \beta(t)$ regularizes (21) can be understood more intuitively as thermodynamical fluctuations (always present in any real physical system unless we freeze it to the absolute zero), with probability 1, kicking instantaneously out any path that happens to go to the points $x$ for which the solution of (18) is non-unique, thereby resolving the ambiguity associated with these positions. The generalized flow can now be defined, 
similarly, as the limit as $k \rightarrow 0$ of the stochastic flow associated with (20), provided this limit is defined in a suitable way. ${ }^{10}$

Mixed limits where both smoothing of the velocity field and Brownian motion are used can be considered as well.

The limiting generalized flows obtained in this manner, however, appear to depend sensitively on the regularization procedure, and they are non-Markov for generic regularizations. The latter fact raises an interesting issue regarding the connection of the Lipschitz indeterministic solutions with the Markov condition.

\subsection{Non-Lipschitz Velocity Fields, Regularizations, and the Markov Condition}

Consider first the following ODE we met in Section 4 (with $\alpha=3 / 4$ corresponding to Norton's original formulation):

$$
\frac{d x}{d t}=\operatorname{sgn}(x)|x|^{\alpha}, x \in \mathbb{R}, \alpha \in(0,1) .
$$

As mentioned before, the set of solutions of this equation is given by

$$
x(t)= \pm\left[(1-\alpha)(t-T)_{+}\right]^{1 /(1-\alpha)},
$$

where $f_{+}=\max (f, 0)$, for an arbitrary $T$.

By resolving the ambiguity of where to map the point $x=0$, the following family of

forward maps $\varphi_{s, t}: \mathbb{R}^{d} \mapsto \mathbb{R}^{d}$ can be constructed:

$$
\varphi_{s, t}^{\omega}(x)=\left\{\begin{array}{ll}
\operatorname{sgn}(x)\left(|x|^{1-\alpha}+(1-\alpha)(t-s)\right)^{1 /(1-\alpha)}, & \text { if } x \neq 0 \\
f^{\omega}(\tau)\left((1-\alpha)(t-\tau)_{+}\right)^{1 /(1-\alpha)}, & \text { if } x=0
\end{array},\right.
$$

\footnotetext{
${ }^{10}$ Typically, the $\varepsilon$-limit is a weaker limit than the $k$-limit in the sense that the regularization by smoothing is more subtle due to the lack of stability of solutions to perturbations and issues with the choice of appropriate convergence. See W. E and Vanden-Eijnden (2003) for more details.
} 
where $\tau=\inf \left(t \geq s: f^{\omega}(s) \neq 0\right)$. Each of the maps $\varphi_{s, t}^{\omega}$ is a weak form of a flow, a quasiflow, satisfying only the following three properties:

(a') $\quad \varphi_{s, s}^{\omega}(x)=x$ for all $s \in[0, T]$;

(b') $\varphi_{s, t}^{\omega}(x)$ is continuous in $s, t, x$;

$\left(\mathrm{c}^{\prime}\right) \quad \varphi_{\tau, t}^{\omega}\left(\varphi_{s, \tau}^{\omega}(x)\right)=\varphi_{s, t}^{\omega}(x)$ for all $x$ and for all $s, \tau, t \in[0, T]$ with $s \leq \tau \leq t$;

By superimposing these quasi-flows and assigning a suitable probability measure a generalized flow can be defined.

In this particular case, the generalized flows, obtained as limits of the standard flows by regularization either via the $k$-limit process or via the $\varepsilon$-limit process, can be shown to be Markov in time. However, as in the next example, Markovianity is not a generic property of generalized flows.

Consider a further generalization of the previous example:

$$
\frac{d x}{d t}=\operatorname{sgn}(x)|x|^{\alpha} g(t), x \in \mathbb{R}, \alpha \in(0,1),
$$

where $g$ is a bounded function. Some solutions of (25) branch at the origin $x=0$ on the time intervals where $g(t)>0$, and other collapse at $x=0$ where $g(t)<0$ (see Fig. 11). 


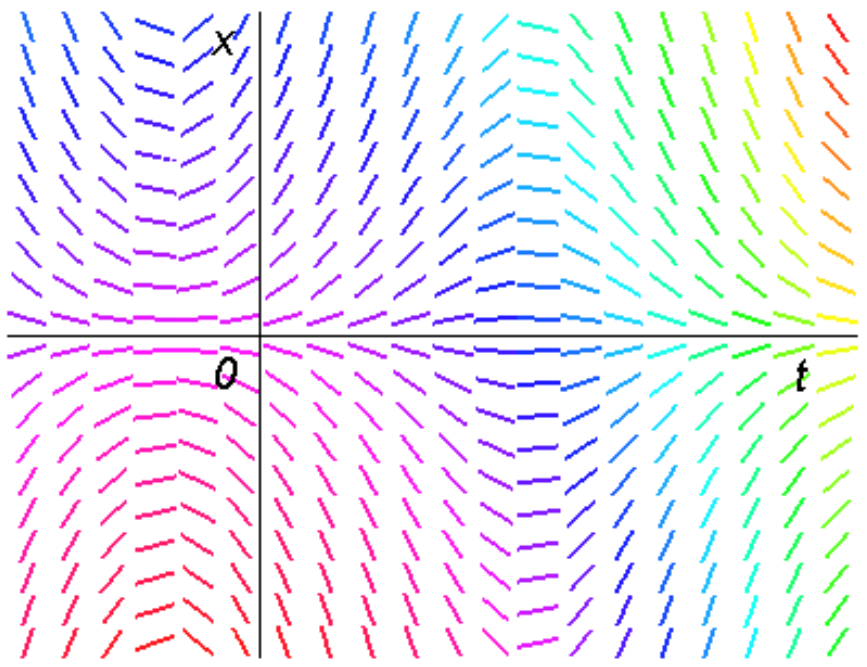

Fig. 11 The set of all solutions of (25) for $\alpha=3 / 4$ and $g(t)=\cos (t)$.

Quasi-flows and the corresponding generalized flow have been properly defined, it can be shown that the generalized flows associated with (25) are not necessary Markovian in time. Though the generalized flows obtained by regularization by the $k$-limit process are Markov, the generalized flows obtained via the $\varepsilon$-limit process are not, so that this feature appears to depend sensitively on the regularization procedure used.

Finally, consider a further relaxation of the condition on the velocity field. In the following ODE the velocity field is continuous in $(x, t)$ but non-Lipschitz at $(x, t)=(0,0)$ and not even Hölder continuous $(x, t)=(0,0)$ :

$$
\frac{d x}{d t}=v(x, t),(x, v) \in \mathbb{R} \times \mathbb{R}
$$

where $v(x, t)$ is given by

$$
v(x, t)= \begin{cases}\frac{2 x}{t}, & \text { if }|x|>t^{2}, \\ 2 \operatorname{sgn}(x) \cdot t, & \text { if }|x| \leq t^{2} .\end{cases}
$$

The set of solutions of (27) can be parameterized as 


$$
x(t)=\operatorname{sgn}(a) t^{2}+a, x(t)=b t^{2}
$$

with $a>0, b \in[-1,1]$ (see Fig. 12):

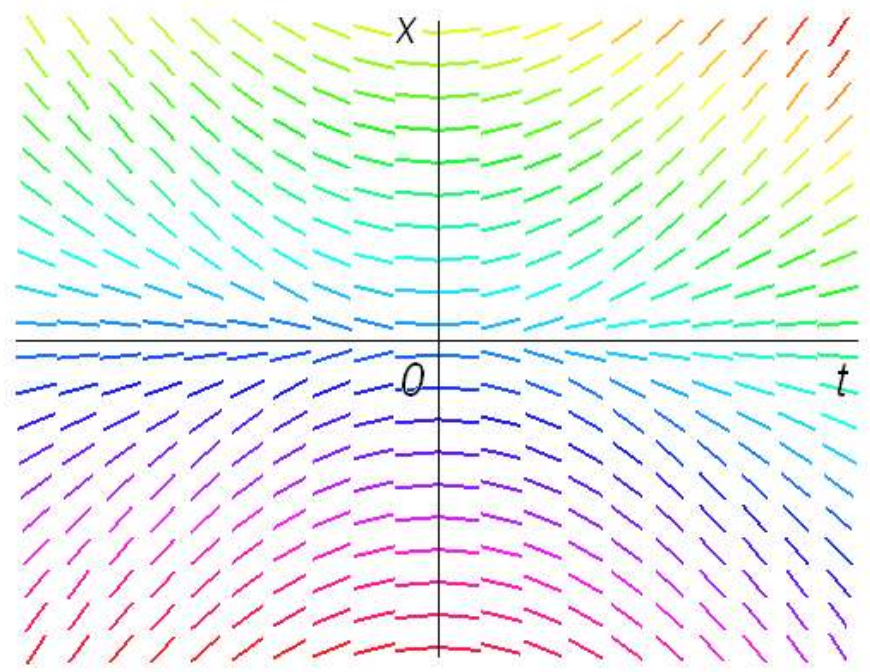

Fig. 11 The set of all solutions of (26).

In this case, the generalized flows associated with (26) can be shown to be nonMarkov in time for both the $k$-limit process flows and most of the $\varepsilon$-limit process flows. The only regularization procedures that do produce a Markov generalized flows in the $\varepsilon$ limit process are those for which, in the limit $\varepsilon \rightarrow 0$, all the paths that collapse on a single node $(0,0)$ exit on a single trajectory.

To summarize the main points of this section, the purpose of drawing these results from fluid dynamics is to illustrate how properties specific to generalized flows associated with first order differential equations with spatially non-Lipschitz right-hand side may lead to interesting and non-trivial features in terms of transport by such fields. Namely, generalized flows, constructed as limits of regularized standard flows, typically appear to lack desirable properties such as stability with respect to perturbations or Markovianity in 
time so that the failure to recognize the Lipschitz condition as an important implicit assumption within Newtonian mechanics may unsurprisingly lead to physically impossible solutions that have no serious metaphysical import, as, for instance, in Norton's causal skeptical anti-fundamentalist program.

\section{Acknowledgments}

I thank Steven Savitt of the Philosophy Department at the University of British Columbia for drawing my attention to the Lipschitz condition, and Alexei Cheviakov of the Mathematics Department at the University of British Columbia for useful discussions.

\section{References}

Arnold, V. I. (1978), Mathematical Methods of Classical Mechanics, New York, Heidelberg, Berlin: Springer-Verlag. Translated from the Russian by K. Vogtmann and A. Weinstein.

Arnold, V. I. (1992), Ordinary Differential Equations, Berlin, Heidelberg, New York, London, Paris, Tokyo, Hong Kong, Barcelona, Budapest: Springer-Verlag. Translated from the Russian by Roger Cooke.

Batterman, R. (2002), The Devil in the Details, Oxford University Press.

Cushing, J. (1990), Theory Construction and Selection in modern Physics: The S-Matrix, Cambridge University Press: Cambridge.

Earman, J. (1986), A Primer on Determinism, Dordrecht, Boston, Lancaster, Tokyo: D. Reidel Publishing Company. 
Eshlami, (1991), Theory of Sensitivity in Dynamical Systems: An Introduction: SpringerVerlag.

Hartmann, S. (2005), "Models and Stories in Hadron Physics", http://philsciarchive.pitt.edu/archive/00002433/

Gilmore, R. (1981), Catastrophe theory for Scientists and Engineers, New York, Chichester, Brisbane, Toronto: John Wiley \& Sons.

Kolmogorov, A.N. (1941), "Dissipation of energy in locally isotropic turbulence", Dokl. Akad. Nauk, SSSR 31, pp. 538-540.

Landau, L.D. and Lifshitz, E.M. (1959), Theory of Elasticity, $3^{\text {rd }}$ ed., Vol. 7 of Course of Theoretical Physics, Transl. from the Russian by J.B. Sykes and W.H. Reid, London, Paris, Frankfurt: Pergamon Press.

Landau, L.D. and Lifshitz, E.M. (1976), Mechanics, $3^{\text {rd }}$ ed., Vol. 1 of Course of Theoretical Physics, Transl. from the Russian by J.B. Sykes and J.S. Bell, Oxford, New York, Toronto, Sydney, Paris, Frankfurt: Pergamon Press.

Laymon, R. (1985), "Idealization and Testing of Theories by Experimentation", in P. Achinstein and Hannaway, eds. Experiment and Observation in Modern Science, Boston: MIT Press and Bradford Books.

Laymon, R. (1995), "Idealizations, Externalities, and the Economic Analysis of Law", in J. Pitt, ed. New Directions in the Philosophy of Technology, Dordrecht: Kluwer Academic Publishers, pp. 185-206.

McMullin, (1985), "Galilean Idealization", Studies in History and Philosophy of Science, $16,247-273$. 
Moulines, C.U. (1996), "Structuralist Models, Idealization, and Approximation", in Hegselmann et al. eds., Modeling and Simulation in the Social Sciences from the Philosophy of Science Point of View (Theory and Decision Theory), Dordrecht: Kluwer Academic Publishers, p. 157-168.

Norton, J. (2003), 'Causation as Folk Science', Philosopher's Imprint, Vol. 3, No. 4, November 2003, http: //www.philosophersimprint.org/003004, to be reprinted in H. Price and R. Corry, Causation and the Constitution of Reality, Oxford University Press.

Redhead, M. (1980), "Models in Physics", British Journal for the Philosophy of Science, 31: $145-163$.

Stroock, D.W. and Varadhan, S.R.S. (1969), "Diffusion Processes with Continuous Coefficients", I and II, Commun. Pure Appl. Math. 22, pp. 345-400.

Stroock, D.W. and Varadhan, S.R.S. (1979), Multidimensional Diffusion Processes, Springer: Berlin.

Weinan, E. and Vanden-Eijnden, E. (2003), "A Note on Generalized Flows", Physica D, 183, pp. 159-174. 